\title{
Hardware Demonstration of Extremely Compact Optical True Time Delay Device for Wideband Electronically Steered Antennas
}

\author{
Betty Lise Anderson, Senior Member, IEEE, James G. Ho, William D. Cowan, Senior Member, IEEE, \\ Olga Blum-Spahn, Allen Y. Yi, Delton J. Rowe, Martin R. Flannery, David L. McCray, Peter Chen, and \\ David J. Rabb
}

\begin{abstract}
An optical true time delay device is demonstrated that is capable of supporting 112 antennas with 81 different delays $(>6$ bits) in a volume $16^{\prime \prime} \times 5^{\prime \prime} \times 4^{\prime \prime}$ including the box with electronics. It uses a free-space design based on the White cell, and alignment is made simple, fast, and robust by the use of slow-tool diamond turning of many optics on a single substrate. Pointing accuracy of the 12 objective mirrors is better than $10 \mu \mathrm{rad}$, and surface roughness is $\approx 45 \mathrm{~nm}$ RMS. Delays vary from 0 to $25 \mathrm{~ns}$ in $312.5 \mathrm{ps}$ increments. Short delays are implemented using delay rods of high refractive index, and long delays using folded mirror trains. Total insertion loss from fiber to detector was $7.82 \mathrm{~dB}$ for the no-delay path, and 10.22 dB for the longest lens train. A three-state tip-style MEMS micromirror array is used to select among the delays, with tilt angles $\pm 1.4^{\circ}$ plus flat, and switching time $<100 \mu$ s for the entire array. An InP wideband optical combiner photodetector array converts the optical signal to RF with $20 \mathrm{GHz}$ bandwidth. The unit survived temperature cycling 0 to $50 \mathrm{C}$ and random vibration on three axes (9.84 $\mathrm{g}$ RMS) with no degradation of signal.
\end{abstract}

Index Terms-Phased arrays, photonic switching systems, optical signal processing, optical delay lines, optical device fabrication.

\section{INTRODUCTION}

$\mathbf{T}$ RUE time delay is preferred over phase shifting for wideband antenna arrays because it avoids beam squint [1]. Optical true time delay (TTD) systems use one light beam for each antenna element in the array, and delay the optical beams individually before demodulating after the delay unit.

Manuscript received December 22, 2010; revised February 25, 2011; accepted March 01, 2011. Date of publication March 10, 2011; date of current version April 20, 2011. Sandia is a multi-program laboratory operated by Sandia Corporation, a Lockheed Martin Company, for the United States Department of Energy's National Nuclear Security Administration under Contract DE-AC04-94AL85000.

B. L. Anderson is with the Department of Electical and Computer Engineering, The Ohio State University, Columbus, OH 43210 USA (e-mail: Anderson@ec.osu.edu).

J. G. Ho, D. J. Rowe, M. R. Flannery, and P. Chen are with Northrop Grumman Aerospace Systems, One Space Park, Redondo Beach, CA 90278 USA (e-mail: james.ho@ngc.com).

W. C. Cowan and O. B. Spahn are with Sandia National Laboratories, Albuquerque NM 87123 USA (e-mail: wdcowan@sandia.gov)

A. Y. Yi and D. L. McCray are with the Department of Integrated Systems Engineering, The Ohio State University, Columbus OH 43210 (e-mail: yi.71@osu. edu).

D. Rabb was with The Ohio State University, Columbus OH 43210 USA. He is now with Wright Patterson Air Force Base, OH 45433 USA (e-mail: david. rabb@wpafb.af.mil).

Color versions of one or more of the figures in this paper are available online at http://ieeexplore.ieee.org.

Digital Object Identifier 10.1109/JLT.2011.2124444
Optical approaches to TTD can be divided roughly into two general approaches, waveguide and free space. Fiber- or waveguide-based approaches switch beams among waveguide paths of varying lengths [2]-[6] or use multiple wavelengths and dispersion to create relative delays [7]-[10]. Fiber lengths can be arbitrarily long and have low loss but the lengths are difficult to control accurately. Fiber switching and coupling loss for a large number of delays can also become substantial. Short delays are better suited to integrated waveguide approaches [11], although the waveguide losses can be an issue. Some fiber approaches use fiber Bragg gratings [12]-[17] in conjunction with tunable lasers, wherein the laser wavelength is tuned to select the grating at the proper distance.

Free-space approaches typically use a series of spatial light modulators to select the paths for arrays of beams [18]-[20] or acousto-optic modulation [21], [22]. The advantage of free space is low weight and equal ease of implementing long and short delays. The drawbacks tend to be a large number of optical components, achieving and maintaining alignment of those components, and large physical size due to beam expansion.

We have previously reported on free-space optical TTDs based on the White cell [23]-[26]. An array of optical beams enters the White cell, and each spot is focused onto a separate pixel of a micro-electromechanical systems (MEMS) array of tilting micromirrors. The micromirror for each beam tilts to select among several choices of path lengths. The spots are subsequently imaged to another section of the same MEMS, and another set of paths is selected.

The White cell can be very compact because 1) many light beams can be processed in parallel with the same hardware; 2 ) the beams can overlap in the same volume without interfering so that the same space is re-used many times; and 3) the device can operate over very wide wavelength range (10 s of $\mathrm{nm}$ ); enabling wavelength division multiplexing.

A disadvantage of the White cell as applied to time delays is that it uses a large number of mirrors. For example, in the optical design used in this paper, there are 18 separate mirrors just in the time delay portion, plus two more ground on the backs of dielectric rods; each of those mirrors has a different tilt angle, and the tilts must be very precisely controlled. Thus, such a system would normally be difficult to align and the alignment difficult to maintain. In this work, we circumvent this problem by fabricating many mirrors on a shared substrate, using slow-tool diamond turning. In this case there are two sets of mirrors, one at each end of the White cell, greatly reducing alignment time and 
TABLE I

Specifications for True Time Delay DeVICE

\begin{tabular}{ll}
\hline \hline Number of input beams & 112 \\
\hline Delay increment $\Delta$ & $312.5 \mathrm{ps}$ \\
\hline Number of delays & 81 \\
\hline Maximum delay & $25.0000 \mathrm{~ns}$ \\
\hline Wavelength & $1.55 \mu \mathrm{m}$ \\
\hline MEMS pixel size & $240 \mu \mathrm{m}$ \\
\hline MEMS pixel pitch & $250 \mu \mathrm{m}$ \\
\hline MEMS tilt angles & $\pm 1.4^{\circ}, 0^{\circ}$ \\
\hline \hline
\end{tabular}

complexity. Such monolithic fabrication approach is also suitable for quantity production.

Table I shows the specifications for this design. There are 112 input channels (to control 112 antenna elements or sub-arrays), with 81 delays ranging from 312.5 ps to $25 \mathrm{~ns}$. The MEMS pixels can tilt to any of three discrete states, "east" or "west" at $1.4^{\circ}$, and "flat" is also a stable position.

This paper is organized as follows. In Section II we give a brief review of the White cell and describe the optical design. Section III discusses the slow-tool diamond turning, and details about the MEMS are given in Section IV. The photodetector array that coherently combines the signals to produce the RF output is described in Section V. Construction and alignment are covered in Section VI, while experimental results are presented in Section VII. Discussion and conclusions are provided in Section VIII.

\section{The White Cell and Optical Design}

The White cell was originally invented in the 1940's for spectroscopy, wherein the absorption of low-pressure gases was measured by causing a single light beam to bounce back and forth multiple times through the sample [27], [28]. Recently the White cell has been adapted to a variety of optical signal processing applications including optical switching [29], optical correlation and quality-of-signal monitoring [30], and optical true time delays for phased array antennas. The detailed operation of these devices is reviewed, for example, in [24], so we will only summarize here.

A basic White cell normally consists of three spherical mirrors, two objective mirrors facing a field mirror, Fig. 1(a). The three mirrors are spherical, with radius of curvature $R$ equal to the distance between them. Light entering the cell is focused to a spot on an input turning mirror, which directs the light to objective mirror $\mathrm{Z} 1$. The light expands on its way to $\mathrm{Z} 1$, and $\mathrm{Z1}$ focuses the beam to a new spot an equal and opposite distance from Z1's center of curvature $(\mathrm{CC}(\mathrm{Z} 1))$. The field mirror, in turn, images the light from Z1 onto Z2. From there the light is focused to a second spot on the field mirror, this time an equal and opposite distance from $\mathrm{CC}(\mathrm{Z} 2)$. The process continues, forming an array of spots on the field mirror, until the beam eventually walks off the field mirror, hits the output turning mirror, and leaves the White cell. The original White cell is a completely passive device, but we replace the spherical mirror in Fig. 1(a) with a MEMS micromirror array and a field lens, producing a new cell that is optically equivalent. The MEMS mirrors, however, can tilt "east" or "west" or remain flat. Thus, when a spot

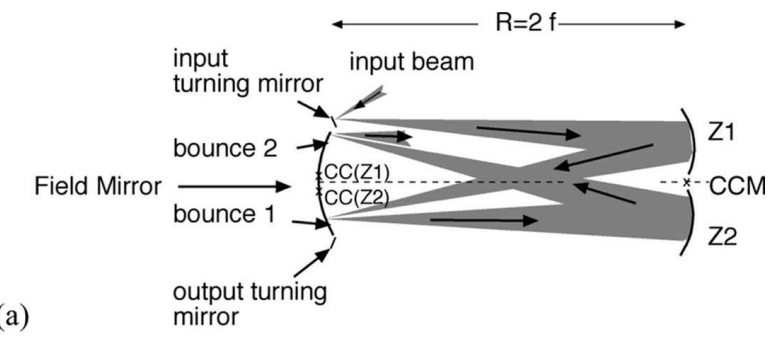

(a)

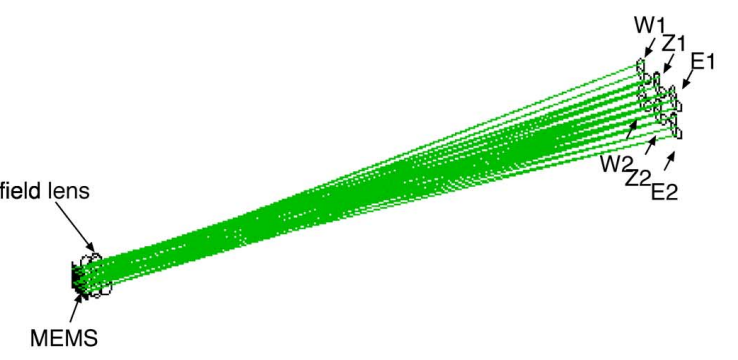

(b)

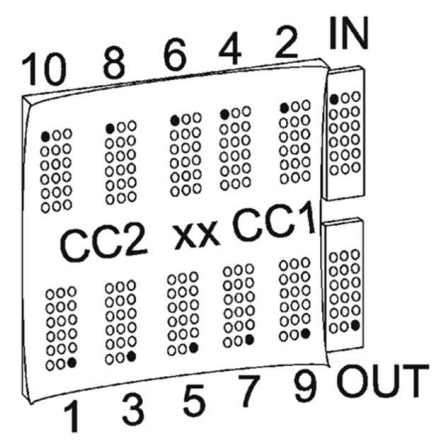

(c)

Fig. 1. (a) Basic White cell. (b) Triple White cell. (c) Resulting spot pattern on the MEMS.

lands on a given MEMS pixel on any particular bounce, it can be directed left or right. In Fig. 1(b), a triple White cell is shown. Mirrors Z1 and Z2 are the objective mirrors of one White cell, and we have added additional objective mirrors E1, E2, W1, and W2. Next, we replace the input beam with an array of input spots (from a fiber array, not shown), which is incident on the input turning mirror, Fig. 1(c). The light from all the beams goes to one of the objective mirrors, which images the array onto a section of the MEMS. The micromirrors can tip East, West, or flat to send each input beam individually to the East, West, or to the null path (Z's). These objective mirrors re-image the array to another section of the MEMS, and the process is repeated. The beams always alternate between upper (Z1, E1, or W1) or lower mirrors. The (images of the) centers of curvature of the upper mirrors all coincide on the MEMS at $\mathrm{CC} 1$, and those of the lower mirrors at CC2. The locations of the CC's are adjusted to produce a given number of bounces on the MEMS, in this case ten. After 10 cycles, the output spots strike an output mirror and go to the detector (not shown).

The number of delays $N$ possible is a function of the number of bounces $m$ and is given by [23]

$$
N=\left(\frac{m-2}{4}+1\right)^{4}
$$




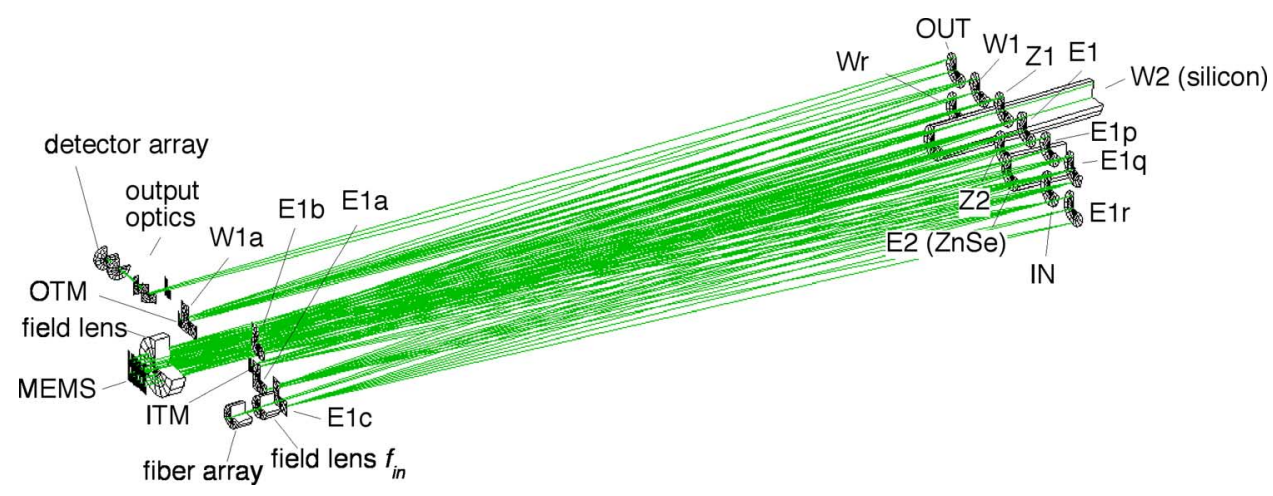

Fig. 2. Quartic White cell design.

Because of the power of four in (1), we call this design a quartic White cell.

To produce the time delays, E1, E2, W1, and $\mathrm{W} 2$ are replaced with delay paths, Fig. 2. The shortest delay path is $\Delta=312.5$ ps, located at E2, and is implemented using a $\mathrm{ZnSe}$ rod. Its back surface is curved and offset from the plane of the objective mirrors to maintain imaging conditions [31]. We allow any given light beam to visit this delay arm up to two times. The next delay arm, $3 \Delta=937.5 \mathrm{ps}$, is implemented in arm $\mathrm{W} 2$, using a silicon rod. ${ }^{1}$ The remaining delays, $9 \Delta=2.8125 \mathrm{~ns}$ and $27 \Delta=8.4375 \mathrm{~ns}$ are realized using folded mirror trains, in arms W1 and E1 respectively. In W1, the light goes from the MEMS to objective mirror W1, then to W1a near the MEMS end of the cell, thence to W1r ("r" for "return") and back through $\mathrm{W} 1 \mathrm{a}$ and $\mathrm{W} 1$ again before returning to the MEMS. Similarly, the longest mirror train consists of E1, E1a, E1p, E1b, E1q, E1c, and E1r, and returns back in the reverse order.

The mirrors W1a, E1a, E1b, and E1c are all on the same plane. We call these the "front four," and all are machined on a single substrate, Fig. 3(a), using slow-tool diamond turning (Section III). These mirrors contain images of the MEMS and they are approximately $6.65 \times 7.6 \mathrm{~mm}$ (there is some demagnification; the MEMS size is $8.0 \times 8.75 \mathrm{~mm}$ ).

Because an image of the MEMS appears on each of the front four mirrors, this was a more convenient place to put the turning mirrors. There is an output turning mirror on W1a, Fig. 3(b), and an input running mirror adjacent to E1a. The turning mirrors must intercept an array of spots without interfering with the adjacent spot array, Fig. 3(c), requiring a very precisely placed straight, sharp edge, hence the term "scraper." Note that the front four mirrors and the scrapers are spherical mirrors, but they all have different pointing angles.

There is a hole in the center of the front-four plate for the beams to pass to and from the MEMS; the field lens can be seen behind it.

Also visible in Fig. 3(a) is a hole for the input beams, which come from a microlensed fiber array. For alignment, an aperture plated with five holes in it was used. The holes correspond to a center beam, and one near each corner of the input array. This allowed for alignment of the fiber array with the MEMS micromirrors. Alignment will be discussed more fully in Section VI.

${ }^{1}$ Silicon was not used for the shortest delay just because it would be too short to handle mechanically.
The front four assembly also anchors the MEMS chip, the field lens, the photodiode-receiver interface, and beam alignment fixtures.

Fig. 4 shows the objective mirrors, also machined from a single substrate. The two delay rods protrude through holes machined into the substrate, and are held and aligned by fixturing behind and attached to the plate. Table II lists the design values for the mirrors and delay rods. All mirrors at the objective end of the White cell are $9.5 \mathrm{~mm}$ in diameter. Mirrors IN, E1, W1, and OUT are slightly toroidal to correct for astigmatism, with $\approx 0.13 \%$ difference between the $x$ and $y$ curvatures. All other mirrors are spherical. The offset for the delay rods is the distance in front of the other mirrors that the rods protrude.

As for the front four, no two objective mirrors have the same pointing angle. Further, the mirrors on the objective end must be aligned with a pointing accuracy better than $10 \mu \mathrm{rad}$ to ensure that all the spots land on the appropriate MEMS pixels on each bounce, as well as on the final detector element in the detector array. The pointing accuracies of the front four mirrors and the MEMS micromirrors are much more forgiving (they can be off by a couple mrad) because their function is to make the beams hit the objective mirrors, which are far larger targets and oversized as well. For this reason the White cell is not sensitive to MEMS mirror pointing angle errors, discussed in Section IV.

The input is a $7 \times 16$ array of single-mode fibers on a 250 $\mu \mathrm{m}$ pitch to match the MEMS pixel pitch. A microlens array maintains the pitch but adjusts the spot size to $60 \mu \mathrm{m}$ radius (1/e power) at the MEMS plane. The MEMS pixels are $240 \mu \mathrm{m}$ in diameter; the spot radius is chosen to be half the mirror radius to keep truncation loss under $0.0004(0.018 \mathrm{~dB})$.

The output optics consist of a folding prism with aspheric front surface and back surfaces, followed by a microlens array that is the same as the input fiber microlens array. It focuses the 112 beams onto a $7 \times 16$ array of InGaP p-i-n photodiodes. The detector array is discussed in Section V.

\section{SLOW-ToOl DiAmond TuRning}

Slow-tool diamond machining is an ultra-precision process in which a complex surface is carved using a single point diamond tool that is used to broach the substrate to remove material. Fig. 5 shows the process. The diamond tool tip rasters across the substrate in $0.5 \mu \mathrm{m}$ steps, while the servo control moves the tool in and out as well as rotates the mirrors. The machine used here is 


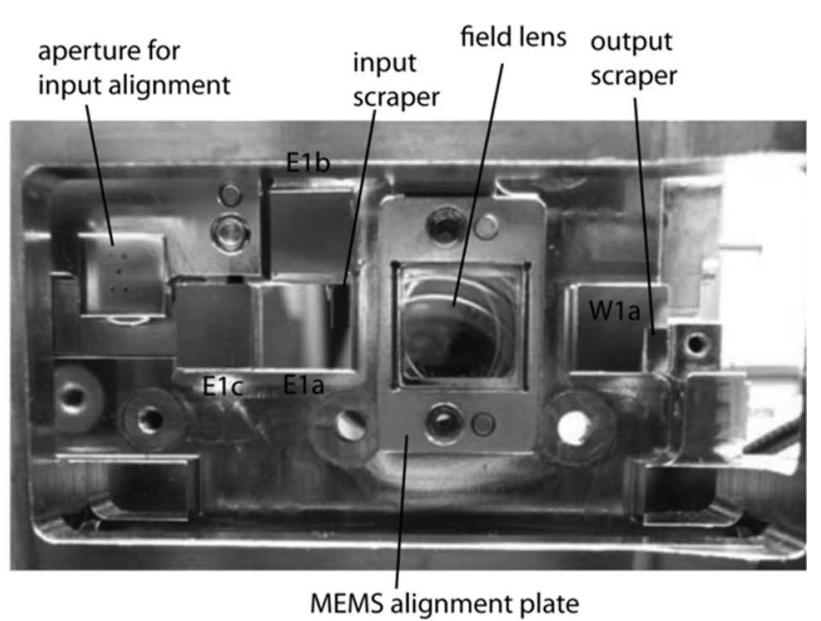

(a)

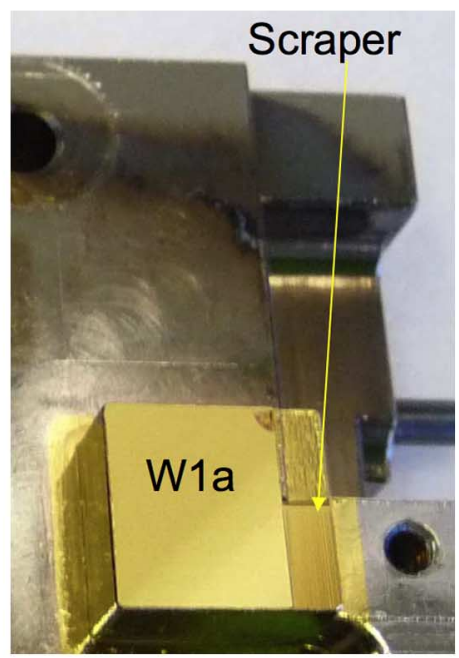

(b)

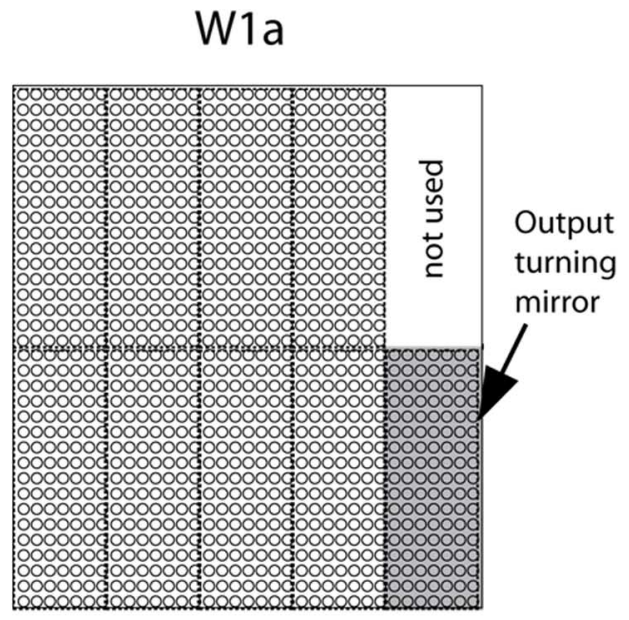

(c)

Fig. 3. (a) Front four mirror unit. (b) Closeup of the output turning scraper mirror. (c) Spot pattern on W1a.

a five axis 350 FG (Freeform Generation) Ultraprecision Machining Center, manufactured by Moore Nanotechnology Inc (Keene, New Hampshire). It uses hydrostatic bearings for both

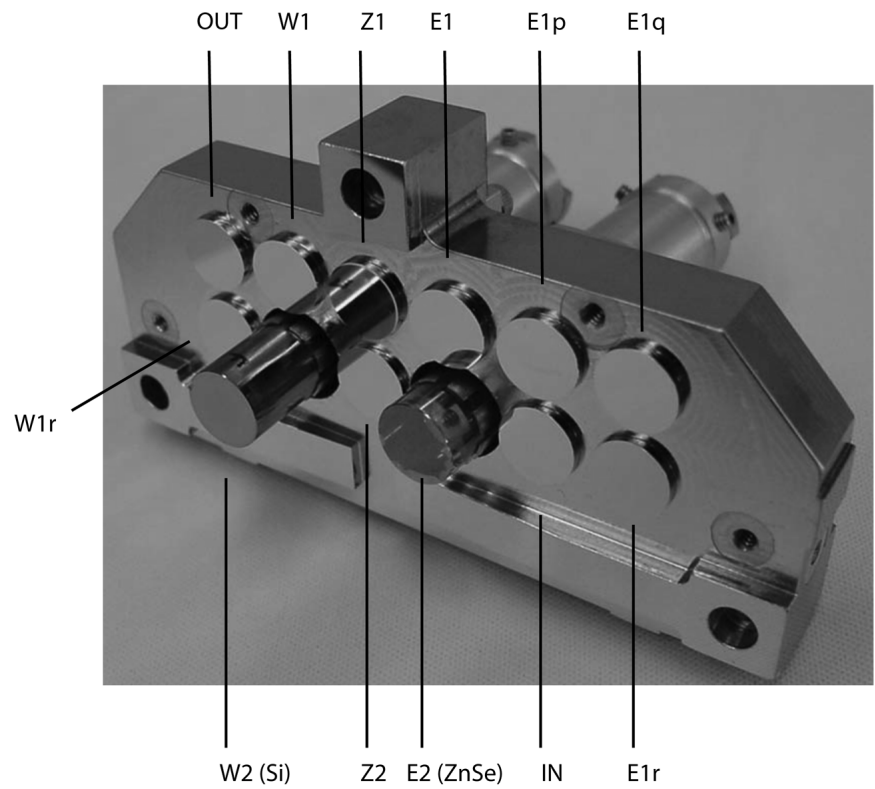

Fig. 4. Objective mirrors.

TABLE II

Design VALUeS OF MiRRoRs (All DiMENSIONS IN MM)

\begin{tabular}{llll}
\hline \hline $\begin{array}{l}\text { Objective End } \\
\text { Mirror }\end{array}$ & Shape & $R_{x}$ & $R_{y}$ \\
\hline $\begin{array}{l}\text { IN, E1, W1, } \\
\text { OUT }\end{array}$ & Toroidal & 221.37 & 221.64 \\
Z1, Z2 & Spherical & 233.11 & -- \\
E1p, E1q, & Spherical & 210.91 & -- \\
E1r, W1r & & & \\
\hline \hline Delay block & Length $L$ & Offset $d$ & Curvature $R$ \\
\hline $\begin{array}{l}\text { Silicon } \\
\text { ZnSe }\end{array}$ & 44.080745 & 12.6752 & 811.23 \\
\hline $\begin{array}{l}\text { Front Four } \\
\text { mirror }\end{array}$ & Shape & $R$ & 573.05 \\
\hline $\begin{array}{l}\text { E1a, E1b, } \\
\text { E1c, W1a, }\end{array}$ & Spherical & 210.93 & -- \\
ITM, OTM & & & \\
\hline \hline
\end{tabular}

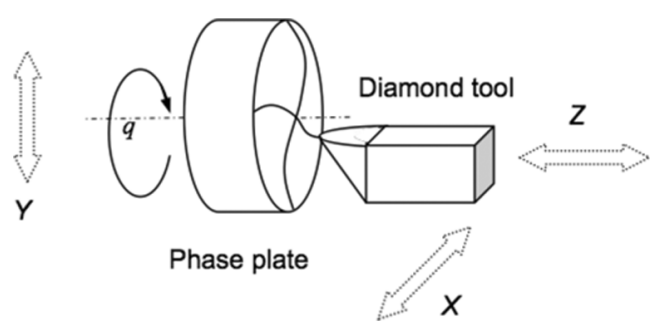

Fig. 5. Principle of the slow-tool diamond machining process.

linear motion and tool station rotation motion. The work spindle uses air bearings to achieve high axial and radial stiffness. The three linear axes are equipped with linear laser-scales capable of resolving $8.6 \mathrm{~nm}$ at a maximum speed of $1800 \mathrm{~mm} / \mathrm{min}$. The work spindle is capable of reaching $6000 \mathrm{rpm}$ (revolutions per minute) while maintaining axial and radial error motion of less than $25 \mathrm{~nm}$. The work spindle can also maintain angular position to less than 0.5 arc second in a modulated mode at speeds up to $2000 \mathrm{rpm}$. The best surface finish the machine can achieve using single point diamond tools are mirror surface finishes of 


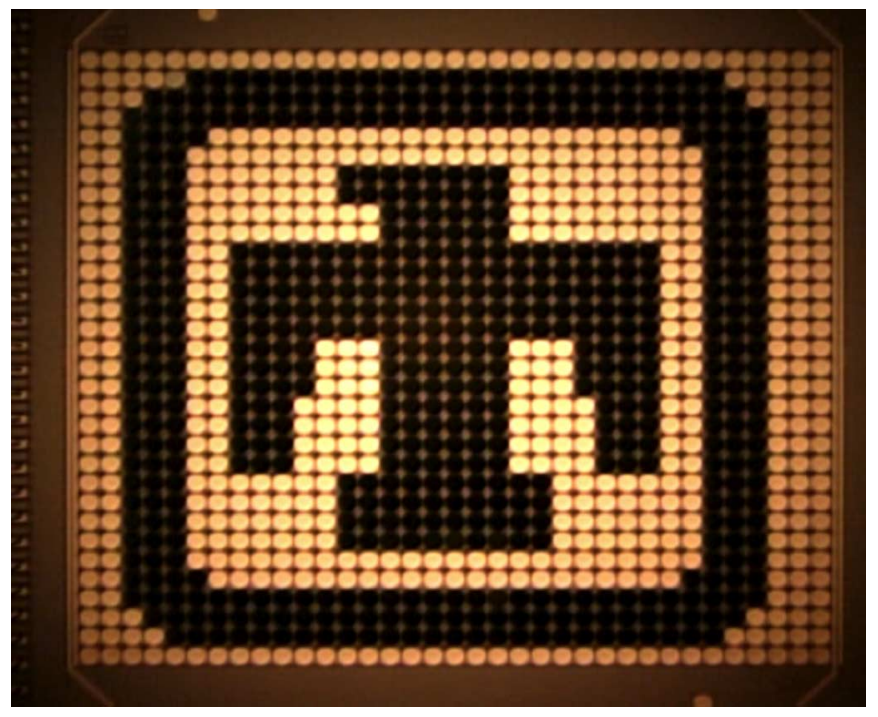

Fig. 6. MEMS is a $32 \times 35$ array of $240 \mu \mathrm{m}$ diameter mirrors.

about 1-2 nm $R_{a}$ (arithmetic average, depending on work piece material).

The mirror blocks were first rough-machined out of superinvar using conventional means, and nickel-plated. The parts were then slow-tool diamond turned to the final dimensions. The diamond turning removes only nickel. The surface finish was measured to have $45 \mathrm{~nm}$ rms roughness. Surfaces were then gold-coated to enhance reflectivity at $1.55 \mu \mathrm{m}$. The curvatures were measured using a Fizeau interferometer against a reference sphere at $633 \mathrm{~nm}$. Curvatures were accurate to within $0.1 \%$ average error. Simulations indicated that pointing accuracy better than $10 \mu \mathrm{rad}$ would be required for the objective mirrors, and this was verified since all the light beams made it through the entire system and landed on the detectors, regardless of delay path.

\section{MEMS}

The MEMS mirror array used in this work was custom-designed for the White cell, and uses a modified SUMMiTTM process with integrated field-effect transistors [32]. The MEMS itself is a $32 \times 35$ array of $240 \mu \mathrm{m}$ diameter mirrors on a 250 $\mu \mathrm{m}$ pitch, Fig. 6 . Each pixel can tip to any of three discrete angles: $\pm 1.4^{\circ}$ and flat. The 2240 electrodes are controlled by a row-column addressing scheme that reduces the pin-out to fewer than 120 wires.

Simulations indicated that the White cell requires MEMS pixel flatness less than $160 \mathrm{~nm}$ across the $240 \mu \mathrm{m}$ mirror. In fact, flatness better than $50 \mathrm{~nm}$ was achieved by careful design of a multi-layer stack that provided the needed stiffness while countering built up stresses. The top layer is aluminum. There are no etch access holes in the metalized mirrors.

The micromirror tip angles are set by an inner and outer set of stops. The outer stops are set by the layout geometry, but the inner stops are created using a timed dimple-etch, which mitigates pattern-density-induced tilt angle variations. Mirror tilts were found to be uniform to within $0.05^{\circ}$ across the array.

The effect of tip angle errors in the MEMS was analyzed. The function of the MEMS pixel tilts is to just to cause the beams to

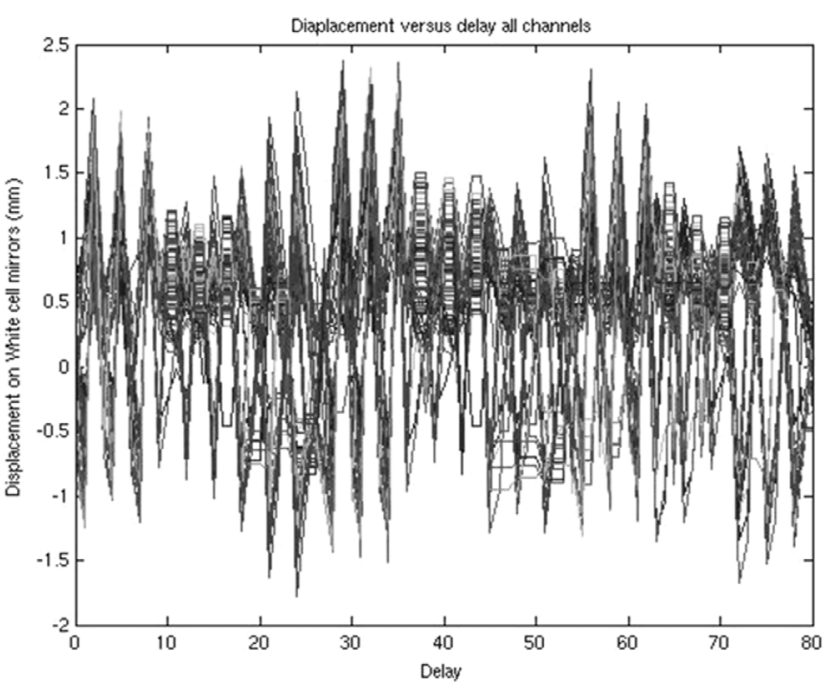

(a)

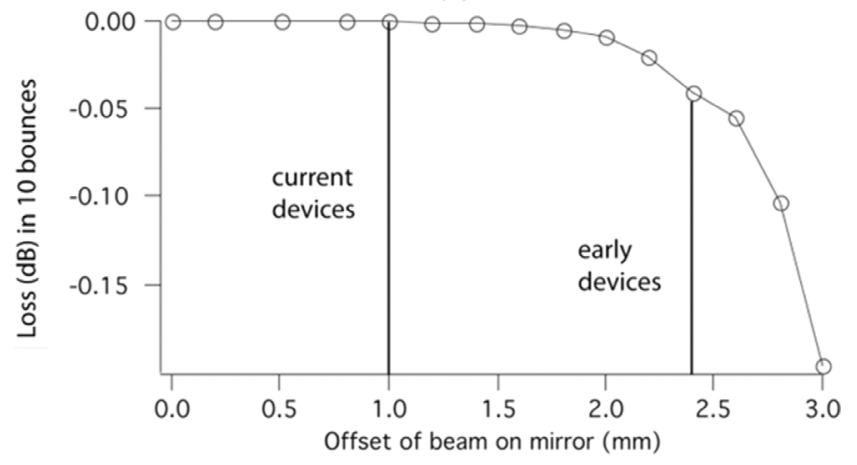

(b)

Fig. 7. (a) Maximum displacement of spots on the objective mirrors for an early MEMS (circa 2006) for all channels, all delays. The maximum displacement then was $2.4 \mathrm{~mm}$, which would incur a truncation loss of $0.04 \mathrm{~dB}$. (b) Loss vs displacement. Current device angle errors are less than $\pm 0.05^{\circ}$, resulting in displacements less than $1 \mathrm{~mm}$, causing negligible loss.

strike the appropriate objective mirror, and the objective mirrors are intentionally oversized. Thus, the pointing angle tolerance does not have to be nearly as tight as the pointing angle of the objectives, which must image one MEMS pixel precisely onto another. Because the image of the spot array is inverted on each successive bounce, the worst-case cumulative error would occur when tilt angle errors alternate sign from one bounce to the next. If a beam were to be truncated by an objective mirror, however, that loss will be sustained only once, since the objective mirrors are images of each other. The truncated beam will simply be replicated on the next mirror, neglecting minor diffraction from the edge and assuming no worse displacements occur later.

Using an early MEMS, circa 2006, we measured the tip angle for every mirror on the array, and calculated the displacement of the beams on the objective mirrors for every channel and for every possible delay. That early device exhibited angle errors from $-0.14^{\circ}$ to $+0.12^{\circ}$, resulting in a maximum displacement of $2.4 \mathrm{~mm}$ on an objective mirror, Fig. 7(a), corresponding to $0.04 \mathrm{~dB}$ loss. More recent devices exhibit maximum tilt errors about one third this size, reducing beam displacement on the objective mirrors to less than $1 \mathrm{~mm}$. Fig. 7(b) shows the loss due to angle errors of this magnitude are negligible. 


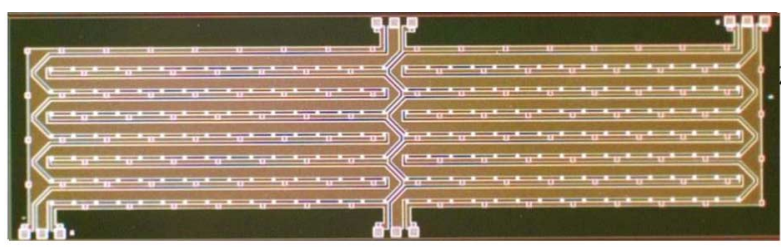

(a)

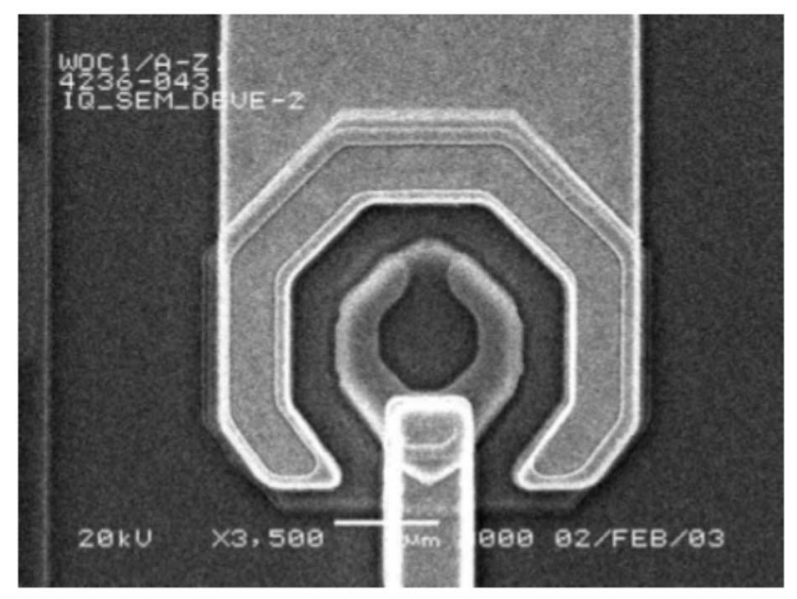

(b)

Fig. 8. (a) $7 \times 32$ array of photodetectors in a traveling-wave coherent combiner. (b) Individual photodetector. The diameter is $9 \mu \mathrm{m}$.

Each mirror array was packaged in a 120-pin ceramic pin grid array using automated die placement to reduce the alignment required after installation of the MEMS into the White cell. The MEMS package was not sealed to prevent Fresnel losses from a window, since the beams pass through it 20 times. Instead, the field lens protects the chip. In future iterations, the field lens would be used to seal the package, with a suitable ambient to dampen the mirror movement appropriately independent of the operating environment.

The MEMS array is driven by an FPGA-based controller that receives mirror position commands via a USB interface from the control PC. The mirror positions are loaded into one of two memory blocks in the FPGA. When the data transfer is completed, the row and column signals are shifted to the other data memory. With the dual data memories, the transfer of new data does not interrupt the mirror refresh signals. Electrode drive signals are sent serially through 16 data lines at a $40 \mathrm{MHz}$ aggregate data rate. Four 32-channel serial-to-parallel converters with high voltage outputs (Supertex HV610) shift the logic level signals to the required Row and Column drive levels (typically 20-25 V).

Measured mirror switching speed for individual mirrors was $<60 \mu \mathrm{sec}$, with the entire array switching in under $100 \mu \mathrm{sec}$.

The functional mirror yield of delivered MEMS arrays was $>99 \%$. Samples of these parts have been life-tested for 10 million cycles, at the end of which two mirrors were stuck.

\section{Photodetector Array}

The receiver consists of a $7 \times 16$ array of dual-depletion InGaAs/InAlGaAs photodiodes on an InP substrate, Fig. 8(a) (a $7 \times 32$ array is shown) [33]. An individual detector is shown in Fig. 8(b). The photodetectors are $9 \mu \mathrm{m}$ in diameter. Arrays of

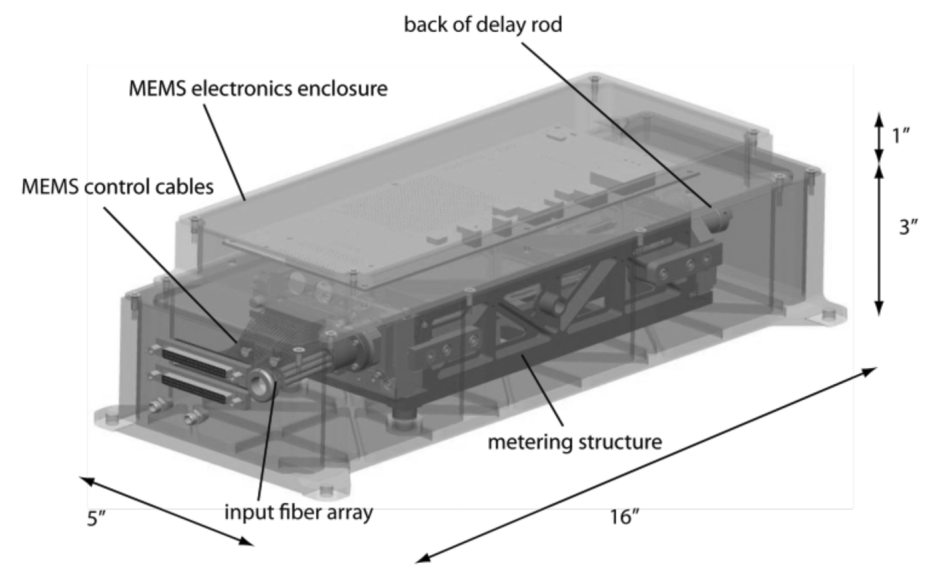

Fig. 9. Entire White cell assembly, including drive electronics, fits in a box $5^{\prime \prime} \times 16 \times 4^{\prime \prime}$.

detectors are connected with an integrated traveling-wave transmission line. The modulated optical signals have pre-set time delays to match the RF propagation time between photodiodes, so that the RF signal is coherently combined within the $20 \mathrm{GHz}$ bandwidth of the receiver. The PD array is packaged together with an integrated microlens array, having lenses with $250 \mu \mathrm{m}$ pitch and $160 \mu \mathrm{m}$ focal length.

\section{CONSTRuCTION AND AlignMent}

By diamond-turning many mirrors on a single substrate, and integrating the microlens arrays with the fibers and detectors, the number of optical components that need to be aligned was reduced from 24 to just 8 . Given that each element requires six degrees of freedom for alignment, this represents a significant simplification. The front four assembly contains, in addition to the four mirror train folding mirrors and input and output scrapers, the input fiber array with its associated microlens and field lens, plus a Risley prism to compensate for tilt, the MEMS and its field lens, and an output folding prism whose entrance and exit surfaces are curved to replace an output field lens. This front-four unit serves as the anchor for everything else. It has alignment pads, also diamond-turned, so that it can be aligned to kinematic interfaces in a metering structure, Fig. 9. Similarly, the objective mirror block with the delay rods mates to the metering structure via a kinematic interface. Thus, once the unit is aligned, either mirror block assembly can be removed and replaced without disturbing the alignment. The positions are repeatable to within $2 \mu \mathrm{m}$. Both the mirror blocks and the metering structure are made of super-Invar for maximum thermal stability.

The objective mirror block, front-four block, and metering structure form a rigid optical assembly with a natural resonance frequency near $510 \mathrm{~Hz}$. This assembly is mounted to the aluminum enclosure at three points with lock pin screws and vibration isolation pads to reduce mechanical coupling and accommodate for thermal expansion. The MEMS electronics are in a separate enclosure attached to the top of the box. The entire assembly is approximately $16 \times 5 \times 4$ inches in volume.

To align the cell, the front-four assembly and the objective mirror block are screwed to the metering structure, and the input fiber array with built-in microlens array and the field lens are 

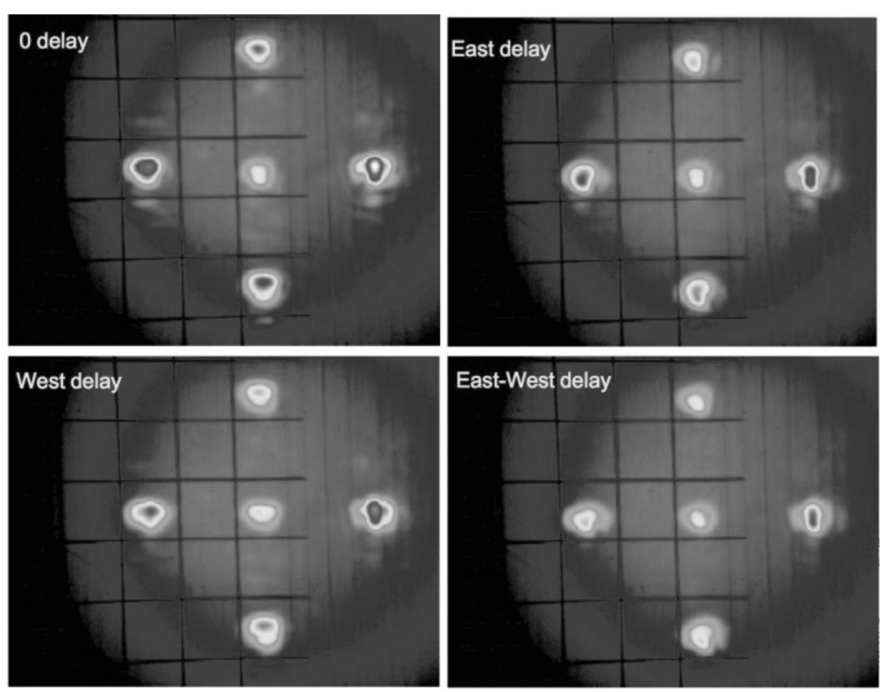

Fig. 10. Spots on the transparent target for a variety of delays.

attached to the front four. There are adjustments for aligning the pointing and position of the input beam array. A small plate with five holes in it, one for each corner and one for the center, is placed in front of the input. To align the input array, the MEMS is replaced by a transparent target plate with a grid pattern on it that marks the positions of the MEMS pixels. The five inputs corresponding to the holes are lighted, and an IR camera is used to image the back of the target.

Once the input array is aligned and fixed in place, the MEMS is installed. The beams can now travel all the way through the White cell (all 10 bounces) and come out toward the receiver. To align the MEMs chip, a second transparent target plate is used at the detector location, also with a grid pattern. The MEMS has control of translation (two directions) and one degree of rotation freedom (clocking) only. Focus is not needed as the beams are collimated between the field lens and the MEMS. One can determine which pixel each of the five test beams is striking by simply toggling the micromirrors and observing when the beams deflect.

Next, the delay rods are aligned by programming the MEMS to send a beam to each rod. Finally, the receiver is aligned by blinking the four corner beams and monitoring the total photocurrent. The receiver position and clocking are adjusted to correctly capture all four corner-beams, and focus is optimized.

Fig. 10 shows a set of spots on the detector grid array as the path through the White cell is varied. It can be seen that there is negligible beam clipping, and that the beam shape remains relatively constant. There is little field distortion, and the spot locations are precise and repeatable, demonstrating that all mirrors curvatures and pointing angles are correct. Further, there is almost no spillover between channels.

\section{EXPERIMENTAL RESULTS}

\section{A. Time Delays}

Time delays were measured using the apparatus of Fig. 11(a). A $1550 \mathrm{~nm}$ laser was modulated with a $1 \mathrm{~ns}$ pulse and then split and coupled into two fibers in the input array. One beam had a manually adjustable reference time delay unit in the path.

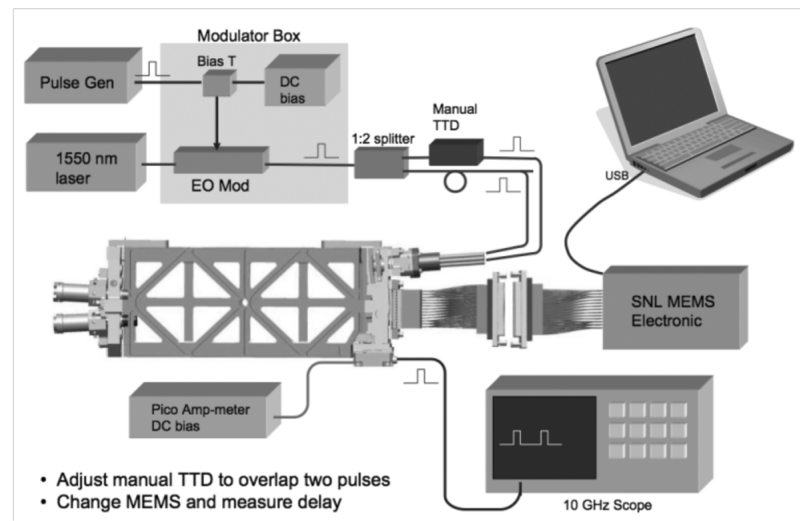

(a)

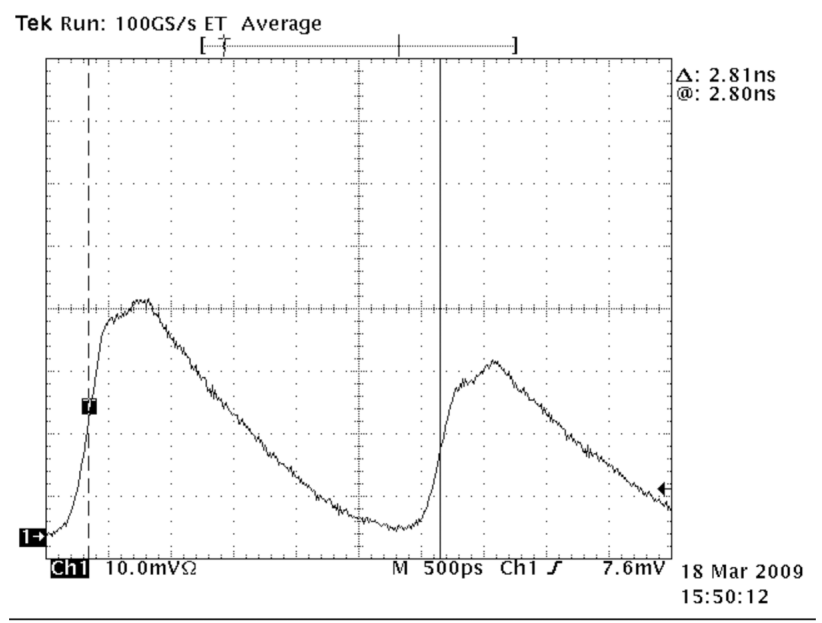

(b)

Fig. 11. Time delay measurements. (a) Apparatus. (b) Sample measurement.

TABLE III

Time Delay Measurements

\begin{tabular}{ccc}
\hline \hline PATH & MEASURED & TARGET \\
ZNSE $(\Delta)$ & 300 PS \pm 10 PS & 312.5 PS \\
SI $(3 \Delta)$ & 940 PS \pm 10 PS & 937.5 PS \\
W1 $(9 \Delta)$ & $2.80 \mathrm{NS} \pm 10$ PS & $2.8125 \mathrm{NS}$ \\
E1 $(27 \Delta)$ & 8.44 NS \pm 10 PS & $8.4375 \mathrm{NS}$ \\
\hline \hline
\end{tabular}

Both beams entered the White cell, and a specific delay was programmed into the other beam. The arrival times are compared on a $10 \mathrm{GHz}$ scope. The picoammeter is used to monitor the DC photocurrent, which provides a monitor on alignment.

Fig. 11(b) shows a sample measurement, in which the beams from two different fibers are detected by the photodiode array. The MEMS is programmed to pass one beam through the null cell, and the other beam passes through the $\mathrm{W} 1$ path for a measured delay of $2.80 \mathrm{~ns} \pm 10 \mathrm{ps}$. This is quite close to the design value of $2.8125 \mathrm{ps}$. Table III gives the delay results for all the other paths. All delays were accurate to within experimental error.

\section{B. Loss}

The losses are divided into two components: White cell insertion loss, measured from the output of the fibers to the entrance to the receiver, and the receiver electro-optic (EO) conversion loss. These two locations are accessible with a power 
TABLE IV

Loss Measurement Averages for the Five InPut Beams Shown IN Fig. 11

\begin{tabular}{|c|c|c|c|c|c|c|c|}
\hline $\begin{array}{l}\text { Delay } \\
\text { path }\end{array}$ & $\begin{array}{l}\text { White } \\
\text { cell } \\
\text { insertion } \\
\text { loss } \\
\text { (dB) }\end{array}$ & $\Delta \mathrm{dB}$ & $\begin{array}{l}\text { Receiver } \\
\text { excess } \\
\text { loss } \\
\text { (dB) }\end{array}$ & $\Delta \mathrm{dB}$ & $\begin{array}{l}\text { Total } \\
\text { inserti } \\
\text { on } \\
\text { loss } \\
\text { (dB) }\end{array}$ & $\begin{array}{l}\# \quad \text { of } \\
\text { mirror } \\
\text { reflect } \\
\text { ions }\end{array}$ & $\begin{array}{l}\text { \# of } \\
\text { rods }\end{array}$ \\
\hline $\begin{array}{l}\text { Null } \\
(0 \Delta)\end{array}$ & 6.6 & & 1.22 & & 7.82 & 24 & \\
\hline $\begin{array}{l}\mathrm{ZnSe} \\
(1 \Delta)\end{array}$ & 6.95 & 0.35 & 1.68 & 0.46 & 8.63 & 23 & 1 \\
\hline $\operatorname{Si}(3 \Delta)$ & 7.58 & 0.97 & 1.44 & 0.22 & 9.02 & 23 & 1 \\
\hline $\begin{array}{l}\text { West } 1 \\
(9 \Delta)\end{array}$ & 8.62 & 2.02 & 1.92 & 0.70 & 10.55 & 28 & \\
\hline $\begin{array}{ll}\text { East } & 1 \\
(27 \Delta) & 1 \\
(27 & \end{array}$ & 8.34 & 1.74 & 1.88 & 0.66 & 10.22 & 36 & \\
\hline $\begin{array}{l}\mathrm{ZnSe}+\mathrm{Si} \\
(4 \Delta)\end{array}$ & 7.95 & 1.35 & 1.83 & 0.61 & 9.78 & 22 & 2 \\
\hline $\begin{array}{l}\text { West } \\
+ \text { East } \\
(36 \Delta)\end{array}$ & 10.51 & 3.91 & 2.43 & 1.20 & 12.94 & 40 & \\
\hline
\end{tabular}

meter; the overlapping of beams in the White cell means one cannot take intermediate loss measurements because the metering structure blocks the path of other parts of the beam. We further divide these losses into inherent losses, set by design and material choice, and excess loss, which depends on the fabrication and alignment quality.

The inherent losses are discussed first. The reflectivity loss of the MEMS dominates due to aluminum micromirrors $(R=$ $0.995)$, which for 10 bounces contributes $-2 \mathrm{~dB}$. The diamondturned mirrors, which are gold-coated, have a reflectivity of 0.965 , and in the null path 13 such surfaces are encountered, contributing another $-2 \mathrm{~dB}$. The total inherent loss for the null delay is estimated to be $-5.6 \mathrm{~dB}$, considering antireflection coatings on the lenses, diffraction, and absorption in the lenses. The receiver EO conversion was measured at $0.5 \mathrm{~A} / \mathrm{W}$.

The excess losses include beam clipping, aberration, and scattering due to misalignment, field curvature, and fabrication defects on mirror surfaces. We observed some beam clipping on the MEMS mirrors and aberration that arises from operating slightly off axis through the three field lenses. Beams in the center $75 \%$ of the array have relatively small variation $(<3 \mathrm{~dB})$ of loss. For beams along the edges of the $7 \times 16$ array, field curvature adds as much as $4 \mathrm{~dB}$ of loss. In the receiver, we estimated an excess loss of about $1.2 \mathrm{~dB}$ for the null path beam. This is most likely due to the combined effect of the very short microlens focal length (160 um) and the small detector area (9 um diameter), resulting in high sensitivity to small tilt error.

Table IV shows measured losses for various delays. These are the averages of the five beams shown in Fig. 12. The figure shows the bounce pattern on the MEMS; the $1 \times 16$ array is re-imaged 10 times in the order shown. The average White cell insertion loss was $6.6 \mathrm{~dB}$, which is about $1 \mathrm{~dB}$ more than the estimated inherent loss, suggesting that alignment losses and mirror defects are well controlled. The EO conversion efficiency was measured at $0.37 \mathrm{~A} / \mathrm{W}, \mathrm{a}-1.2 \mathrm{~dB}$ change from the prediction. This is attributed to accumulated aberrations, mostly focusing error. The total insertion loss for the null path was $7.82 \mathrm{~dB}$, under the design target of $8 \mathrm{~dB}$.

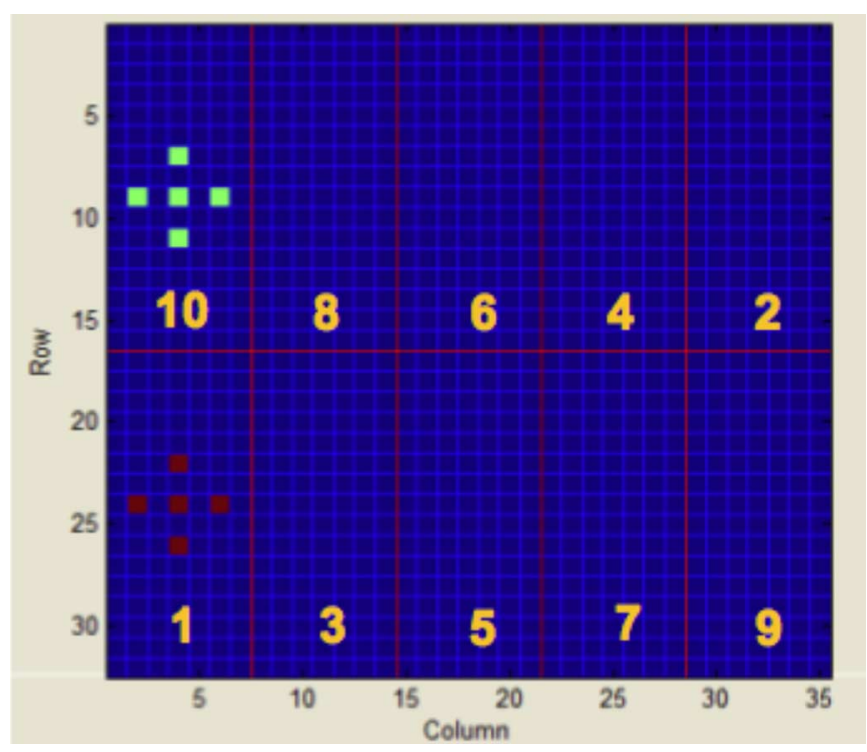

Fig. 12. Five spots used for the loss measurement of Table IV.

As the delay changes, the path and thus the number and nature of surfaces also changes. The average loss per mirror in the East mirror train (13 extra mirror surfaces) was $0.2 \mathrm{~dB}$ (the longer mirror train) but $0.7 \mathrm{~dB}$ per mirror for the short mirror train (five extra surfaces). The reason for the difference is not clear, but may be due to either surface curvature or mirror pointing error in either W1 or W1r. The rods incur both reflection and absorption loss; the $\mathrm{ZnSe}$ (short) rod added $0.35 \mathrm{~dB}$ of loss and the Si (long) rod added $0.97 \mathrm{~dB}$. Also note that as the delay path changes the wavefront aberrations also change, contributing some variation in the receiver excess loss.

\section{Thermal Cycle and Shock}

The unit was thermal-cycled in dry ambient air, between $0 \mathrm{C}$ and $50 \mathrm{C}$ with 1-hour dwell times at each extreme. Four cycles were completed with 3 degrees $\mathrm{C} / \mathrm{min}$ transition rates. That was 

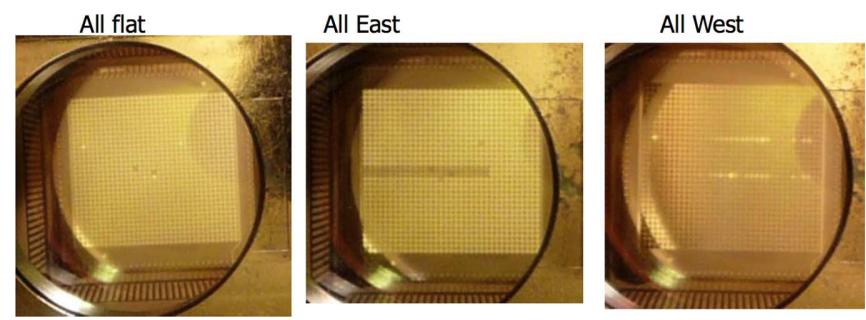

Fig. 13. MEMS pixels stuck after vibration test.

TABLE V

Photodector Currents Pre- and Post-Vibration for Each AXes, With All Pixels Flat (No BeAm ARRives at DeteCtor) and With PatTerns APPLIED (BEAMS ARRIVE AT PHOTODETECTORS)

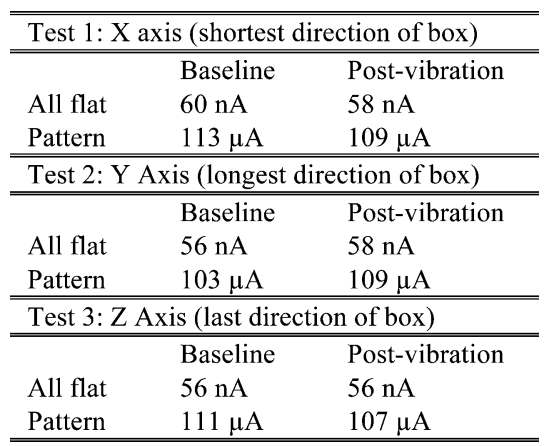

followed by random vibration, $9.84 \mathrm{~g} \mathrm{rms}$, for 180 second duration on each of three axes. Post-test evaluation included visual inspections for any loose components, verification of MEMS operation, and check of alignment and insertion losses.

After vibration on the first axis (shortest dimension of the box), two lid fasteners were found to be loose. They were tightened, and did not loosen again during the remaining two axes of vibration.

After all three axes of vibration, the MEMS was examined, and a few mirrors were found to have become stuck, Fig. 13. When all mirrors were programmed to turn East or West improper operation of 4 rows was observed. Similar failures observed in MEMS testing were found to be attributable to shorting of stuck mirrors to underlying wiring. This particular MEMS part had been in use in lab air for over a year and survived a previous vibration (and unintentional shock) test that most certainly generated debris in the optical assembly, thus these results are encouraging. The part continued to function normally in unaffected regions thereby allowing post-vibration optical testing to continue.

To evaluate any changes in alignment or loss due to thermal cycling and vibration, the photodetector current was monitored for the MEMS pixels all flat (a condition that would not deliver any beams to the photodectors) and for a particular MEMS pattern that did direct beams to the output after navigating all 10 bounces in the White cell. Table $\mathrm{V}$ shows negligible change in the currents pre- and post-vibration.

From fiber to fiber, about $1.6 \mathrm{~dB}$ spread of variation for $90 \%$ of the fibers was observed. In the remaining $10 \%$, the maximum. difference was $-4 \mathrm{~dB}$ for one channel relative to the average insertion loss.

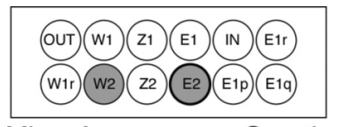

MirrorArrangement Quartic ( 81 delays in 10 bounces)

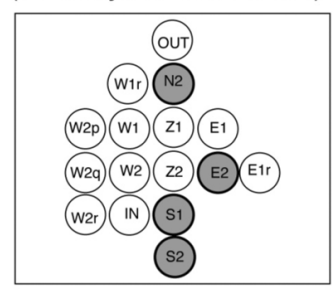

MirrorArrangement in Octic (6,399 delays in 18 bounces)

Fig. 14. White cell (objective) mirror for quartic versus octic cell. Shaded mirrors represent delay rods.

\section{DISCUSSION AND CONCLUSIONS}

The White cell described here is "quartic" and only requires the MEMS mirrors to tip to one of three states. An "octic" cell has been proposed elsewhere [25], [34] in which the number of delays goes as $\gamma^{8}$ instead of $\gamma^{4}$, where $\gamma$ is the base of the counting system (in the quartic cell here, we counted in base 3 , hence $\gamma=3$ ). An octic cell can provide 6399 delays in 18 bounces, essentially doubling the number of bits of resolution from over 6 to over 12. It should be noted that higher number of bounces for the octic cell tightens the mirror pointing precision requirement. Fig. 14 compares the objective mirror arrangement in the current quartic cell to a notional octic cell. This design uses additional delay paths to the north and south, and has four delays rods instead of two. Note, however, that the structure of the MEMS required is no more complex. That is because half of the MEMS mirrors need to tip only east, west, and flat, and the other half tip only north, south, and flat, so only a different mask is required. The rest of the White cell would be exactly the same as the quartic cell. This fact, plus the simplicity of the diamond turning, means that an octic cell would not be much more difficult to fabricate than the quartic cell.

This demonstration establishes the feasibility of fabricating a robust, compact, light-weight White cell device despite the complexity, number of mirrors, and requirement for high-precision pointing angles. By combining many mirrors on a single substrate, and using high-precision slow-tool diamond machining to create them, the otherwise daunting task of aligning all the optics individually is reduced to aligning just eight major optical components. Once aligned, the unit can be disassembled and reassembled without loss of alignment. There are 1120 MEMS micromirrors, 19 additional imaging mirrors (each with a different pointing angle), 227 lenses (including 112 in each of the two microlens arrays), 112 photodetectors and 112 input fibers. There are, in this design, about 820 mechanical degrees of freedom, and approximately 2145 optical tolerances. By comparison, the number of optical tolerances in the James Webb Space Telescope (telescope only) is about 380 and for the Hyperion Hyperspectral Spectrometer (including polarization) is about 213. The slow-tool diamond turning process makes 
possible fabrication of systems as complex as the White cell because it is versatile and precise enough to create optical-quality mirrors while simultaneously producing alignment pads for ultra-precise and instant assembly.

To our knowledge, this optical implementation of RF true time delay represents the most hardware compressive and lowest loss approach ever demonstrated.

\section{REFERENCES}

[1] , H. Zmuda and E. N. Toughlian, Eds., Photonic Aspects of Modern Radar. Norwood, MA: Artech House, 1994, The Artech House Optoelectronics Library.

[2] A. P. Goutzoulis and D. K. Davies, "Hardware-compressive 2-D fiber optic delay line architecture for time steering of phased-array antennas," Appl. Opt., vol. 29, pp. 5353-5359, 1990.

[3] O. Raz et al., "Submicrosecond scan-angle switching photonic beamformer with falt RF response in the C and X bands," J. Lightw. Technol., vol. 26, no. 10, pp. 2774-2781, Oct. 2008.

[4] R. Taylor and S. Forrest, "Steering of an optically-driven true-time delay phased-array antenna based on a broadband coherent WDM architecture," IEEE Photon. Technol. Lett., vol. 10, no. 1, pp. 144-146, Jan. 1998.

[5] N. Madamopoulos and N. Riza, "All-fiber connectorized compact fiber optic delay line modules using three-dimenstional polarization optics," Opt. Eng., vol. 39, pp. 2338-2344, Sep. 2000.

[6] J.-D. Shin et al., "Optical true time delay feeder for X-band phased array antennas composed of $2 \times 2$ optical MEMS switches and fiber delay lines," IEEE Photon. Technol. Lett., vol. 16, no. 5, pp. 1364-1366, May 2004.

[7] O. Raz et al., "Implementation of photonic true-time delay using highorder-mode dispaersion compensating fibers," IEEE Photon. Technol. Lett., vol. 16, no. 5, pp. 1367-1369, May 2004.

[8] R. D. Esman et al., "Two optical-control techniques for phased array: Interferometric and dispersive-fiber true time delay," Trans. Opt. Process. Into Syst., pp. 133-143, 1993.

[9] M. Y. Frankel and R. D. Esman, "Dynamic null steering in an ultrawideband time-steered array antenna," Appl. Opt., vol. 37, pp. 5488-5494, 1998.

[10] H. Zmuda et al., "A photonic implementation of a wideband nulling system for phased arrays," IEEE Photon. Technol. Lett., vol. 10, no. 5, pp. 725-727, May 1998.

[11] B. Kanack et al., "Optical time delay network for phased arrays," Trans. Opt. Process. Into Syst., pp. 114-132, 1993.

[12] J. Yang et al., "Continuous true-time-delay beamforming employing a tunable multiwavelength fiber ring laser source with euqally increased or decreased spacing," Opt. Eng., vol. 42, pp. 239-244, Jan. 2003.

[13] P. Q. Thai et al., "A novel simploified dual beam-former using multichannel chirped fiber grating and tunable optical delay lines," J. Lightw. Technol., vol. 26, no. 10, pp. 2629-2634, Oct. 2008.

[14] D. A. Cohen et al., "Optically controlled serially fed phased array sensor," IEEE Photon. Technol. Lett., vol. 8, no. 12, pp. 1683-1685, Dec. 1996.

[15] J. Medberry and P. Matthews, "Investigations of linearly chirped fiber Bragg gratings for time-steered array antennas," Fiber and Integrated Optics, vol. 19, pp. 469-482, 2000.

[16] Y. Liu et al., "Continuous true-time-delay bemaforming for phased array antenna using a tunable chirped fiber grating delay line," IEEE Photon. Technol. Lett., vol. 14, no. 8, pp. 1172-1174, Aug. 2002.

[17] D. B. Hunter et al., "Demonstration of a continously variable true-time delay beamformer using a multichannel chirped fiber grating," IEEE Trans. Microw. Theory Tech., vol. 54, no. 2, pp. 861-867, Feb. 2006.

[18] N. A. Riza, "Liquid crystal-based optical control of phased array antennas," J. Lightw. Technol., vol. 10, no. 12, pp. 1974-1984, Dec. 1992.

[19] H. R. Fetterman et al., "Optically controlled phased array radar receiver using SLM switched real time delays," IEEE Microw. Guided Wave Lett., vol. 5, no. 11, pp. 414-416, Nov. 1995.

[20] D. Dolfi and P. Joffre, "Experimental demonstration of a phased-array antenna optically controlled with phase and time delays," Appl. Opt., vol. 8, pp. 1824-1828, 1996.
[21] K. Wagner et al., Wide-band all-optical BEAMTAP 2001.

[22] R. J. Berinato, "Acousto-optic tapped delay line filter," Appl. Opt., vol. 32, pp. 5797-5809, Oct. 10, 1993.

[23] B. L. Anderson and R. Mital, "Polynomial-based optical true-time delay devices using MEMs," Appl. Opt., vol. 41, pp. 5449-5461, Sep. 10, 2002.

[24] C. M. Warnky et al., "Demonstration of a quartic cell, a free-space true-time-delay device based on the white cell," J. Lightw. Technol., vol. 24, no. 10, pp. 3849-3855, Oct. 2006.

[25] R. Mital et al., "Design and demonstration of a higher-order polynomial cell-Octic cell," J. Lightw. Technol., vol. 24, no. 2, pp. 982-990, Feb. 2006.

[26] B. L. Anderson et al., "Binary optical true tieme delay based on the white cell: Design and demonstration," J. Lightw. Technol., vol. 24, no. 4, pp. 1886-1895, Apr. 2006.

[27] J. U. White, "Very long optical paths in air," J. Opt. Soc. Amer., vol. 66, pp. 411-416, 1976.

[28] J. White, "Long optical paths of large aperture," J. Opt. Soc. Amer., vol. 32, pp. 285-288, 1942.

[29] B. L. Anderson et al., "Optical cross-connect switch based on tip/tilt micromirrors in a white cell," IEEE J. Sel. Topics Quantum Electron., vol. 9, no. 2, pp. 579-593, Mar./Apr. 2003.

[30] B. L. Anderson et al., "Real-time all-optical quality of service monitoring using correlation and a network protocol to exploit it," Appl. Opt., vol. 43, pp. 1121-1130, Nov. 5, 2003, Accepted.

[31] S. Kunathikom et al., "Design of delay elements in binary optical true-time delay device using a white cell," Appl. Opt., vol. 42, pp. 6984-6994, Dec. 2003.

[32] W. D. Cowan et al., "Integrated FET-Polysilicon micromachining process for optical MEMS," in Proc. IEEE CLEO, 2006, pp. 64-65.

[33] J. G. Ho et al., "Wideband coherent combining of photonic RF signals with photodiode array," presented at the GOMAC Tech, Monterey, CA, 2004.

[34] B. L. Anderson et al., "The "Octic" white cell true-time delay device," presented at the GOMAC Government Microcircuits Applications Conf., Tampa, FL, 2003.

Betty Lise Anderson (M'78-SM'95) obtained the B.S. degree in electrical engineering at Syracuse University, Syracuse, NY, in 1978, and the M.S. and Ph.D. degrees in materials science and electrical engineering at the University of Vermont, Burlington, in 1988 and 1990, respectively.

She spent nine years in industry, including at Tektronix, Inc., GTE Laboratories, and Draper Laboratories. She is now a professor at The Ohio State University in the Department of Electrical and Computer Engineering. Her current research interests include analog optical signal processing, devices for optical communication systems, coherence, and semiconductor devices. She is the coauthor (with Richard L. Anderson) of Fundamentals of Semiconductor Devices (McGraw Hill, 2005).

Prof. Anderson is a Senior Member of the Optical Society of America and a member of the American Society for Engineering Education. She received the Outstanding Woman in Technology Award from TechColumbus in 2006.

William D. Cowan (S'78-M'82-SM'03) received the B.S. degree in electrical engineering from the West Virginia Institute of Technology, Montgomery, WV, in 1982. He received the M.S. degree in electrical engineering in 1984, and the $\mathrm{Ph} . \mathrm{D}$. degree in electrical engineering in 1998, both from the Air Force Institute of Technology, Wright-Patterson Air Force Base, $\mathrm{OH}$.

Recently retired from the Air Force, he has over 25 years of experience in defense-related research and development. He has been working on micromirrors and adaptive optics since 1995 . He has authored more than 38 research papers in a number of diverse technical fields including; RF components, high temperature superconductors, high power electromagnetic effects testing, long wavelength infrared detection, adaptive optics, and MEMS. He has also written two book chapters on micromirrors for adaptive optics. Dr. Cowan joined Sandia National Laboratories in 2003 and is a Principal Member of Technical Staff in the RF Microsystems Department.

Dr. Cowan is a member of SPIE, Tau Beta Pi, and Eta Kappa Nu. 
Olga Blum Spahn received the B.S. degree from the University of Illinois, Champaign-Urbana, in 1987, and the M.S. and Ph.D. degrees from the University of California, Berkeley, in 1990 and 1992, respectively, all in electrical engineering.

In 1993 she joined Sandia National Laboratories, Albuquerque, NM. Her research interests include MEMS/photonics integration, including post-processing, process compatibility and fabrication. Additionally, she is involved in optical microsystem design, fabrication and testing. Her other interests include compound semiconductor-based monolithic integration of MEMS and lasers/detectors. She has over 100 publication and conference presentations, including several invited talks and book chapters.
David J. Rabb received the B.S. degree in electrical engineering from Ohio University, Athens, in 2002, and the M.S. and Ph.D. degrees in electrical and computer engineering from the Ohio State University, Columbus, in 2005 and 2008 , respectively.

$\mathrm{He}$ is currently at Wright Patterson Air Force Base, OH. 